\title{
Clock gene expression in human and mouse hepatic models shows similar periodicity but different dynamics of variation
}

Gianluigi Mazzoccoli, Rosa Rubino, Cristiana Tiberio, Francesco Giuliani, Manlio Vinciguerra, Jude Oben, Angelo De Cata, Roberto Tarquini, Salvatore De Cosmo, Shu Liu \& Yanning Cai

To cite this article: Gianluigi Mazzoccoli, Rosa Rubino, Cristiana Tiberio, Francesco Giuliani, Manlio Vinciguerra, Jude Oben, Angelo De Cata, Roberto Tarquini, Salvatore De Cosmo, Shu Liu \& Yanning Cai (2016) Clock gene expression in human and mouse hepatic models shows similar periodicity but different dynamics of variation, Chronobiology International, 33:2, 181-190, DOI: 10.3109/07420528.2015.1132722

To link to this article: http://dx.doi.org/10.3109/07420528.2015.1132722

曲 Published online: 15 Mar 2016.

Submit your article to this journal ¿

Џ Article views: 6

Q View related articles $₫$

View Crossmark data 5 


\title{
Clock gene expression in human and mouse hepatic models shows similar periodicity but different dynamics of variation
}

\author{
Gianluigi Mazzoccoli (1)a, Rosa Rubino ${ }^{a}$, Cristiana Tiberio ${ }^{a}$, Francesco Giuliani ${ }^{b}$, Manlio Vinciguerra ${ }^{\mathrm{a}, \mathrm{c}, \mathrm{d}, \mathrm{e}}$,

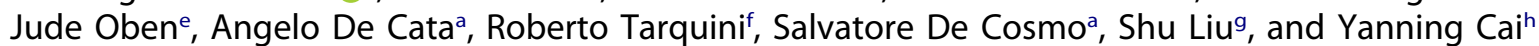

\begin{abstract}
aDivision of Internal Medicine and Chronobiology Unit, IRCCS Scientific Institute and Regional General Hospital "Casa Sollievo della

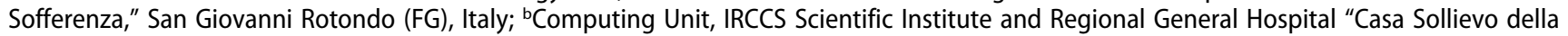

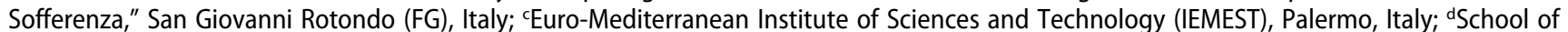
Science and Technology, Nottingham Trent University, Nottingham, United Kingdom; eUniversity College London, Institute for Liver and Digestive Health, Division of Medicine, Royal Free Campus, London, United Kingdom; fDepartment of Clinical and Experimental Medicine,

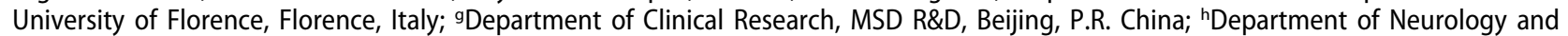
Neurobiology, Xuanwu Hospital of Capital Medical University, Key Laboratory for Neurodegenerative Diseases of Ministry of Education, Beijing, P. R. China
\end{abstract}

\begin{abstract}
The biological hard-wiring of 24-hour rhythmicity relies on the circadian clock circuitry, made of peripheral oscillators operated by molecular clockworks and synchronized through humoral and neural outputs by central oscillators located in the hypothalamic suprachiasmatic nuclei. Metabolically active tissues, such as the liver, are entrained also by local cues represented by metabolic flux related to feeding. The mechanics of the molecular clockwork have been explored by studies using cell lines and wild type or genetically engineered mouse models. There is a compelling need to reduce the use of animals in experimental settings. The aim of our study was to evaluate the periodicity and dynamics of functioning of the hepatic clock gene machinery in human and mouse hepatic models. We compared the results obtained in human hepatoma cells (HepG2 cells) and in mouse liver, and a significant 24-hour rhythmic component was found for five clock genes in the HepG2 cells (Bmal1, Cry1, Per1, Per2, NR1D1) and for six clock genes in the mouse liver (Bmal1, Clock, Cry1, Per1, Per2, NR1D1). The amplitude of oscillation rendered by the cosine curve and the dynamics of expression rendered by the rate of change (the derivative of gene expression level with respect to time) were greater in the mouse liver than in the HepG2 cells for Bmal1, Per1, Per2 and NR1D1, and the cosine curve phase was different for many of them. In conclusion, the periodicity of expression of the clock genes showed similar patterns when the two experimental models were compared, whereas the dynamics of transcription in human hepatoma cells cultured in vitro were less vigorous and phased in a different way when compared to mouse hepatic tissue. The results support the reliability of the human hepatic in vitro model as an alternative to animal models only to study the periodicity of function of the molecular clockwork, but not to evaluate the dynamics of clock gene expression.
\end{abstract}

\section{KEYWORDS}

Chronobiology; clock genes; hepatocytes; liver

\section{Introduction}

Cell, tissue and organ functions are characterized by nycthemeral oscillations with approximately 24-hour periodicity, defined circadian. The rhythmicity is generated by sub-cellular molecular clockworks identified in virtually every tissue investigated up until now (Bell-Pedersen et al., 2005; Mazzoccoli et al., 2011; Mazzoccoli et al., 2012a). In mammals, the molecular clocks ticking in every single cell are synchronized by the circadian timing system (CTS) that orchestrates the array of rhythms in cellular and organismal physiology (Liu et al., 2007).

The CTS comprises biological clocks in the hypothalamic suprachiasmatic nuclei entrained to the environmental light/dark cycle by photic inputs conveyed by the retino-hypothalamic tract, and synchronizing self-sustained oscillators in peripheral tissues through humoral and nerve outputs (Herzog, 2007; Mazzoccoli, 2011). The cellular oscillator consists of a molecular clockwork operated by the circadian genes Arntl (also

CONTACT Gianluigi Mazzoccoli g.mazzoccoli@operapadrepio.it Department of Medical Sciences, Division of Internal medicine and Chronobiology Unit, IRCCS Scientific Institute and Regional General Hospital "Casa Sollievo della Sofferenza," San Giovanni Rotondo (FG), 71013 Italy. Tel: +39 0882410255. 
called Bmal1), Clock, Per 1-3, Cry 1-2, NR1D1 (encoding Rev-erba) and Rora (encoding RORa) (Nagoshi et al., 2004). Bmal1 and Clock encode transcription factors that form a heterodimer to bind the E-boxes in the promoter region of Per 1 , Per2, Per3, as well as Cry 1 and Cry 2 genes, activating their expression and the synthesis of the encoded proteins. Per and Cry proteins in the cytoplasm complex and then return in the nucleus, where they inhibit Clock:Bmall transcriptional activity, closing a feedback loop of transcription and translation that completes a cycle in approximately 24 hours (Takahashi et al., 2008). Clock:Bmall drives also the expression of the nuclear receptors Rev-erba and Rora, which in turn influence Bmall expression in a negative and positive manner, respectively, fueling amplitude and robustness of oscillation in the biological clock (Cho et al., 2012; Mazzoccoli et al., 2012b). Phosphorylation, acetylation, ubiquitination and sumoylation of proteins encoded by the clock genes play a key role in the functioning of the molecular clockwork (Agostino et al., 2009; Duguay \& Cermakian, 2009; Eide et al., 2002). The clock gene machinery drives the expression of transcription factors, clock-controlled genes expressed in all tissues and tissuespecific output genes, which manage cellular and tissue functions such as proliferation, apoptosis, autophagy, DNA repair and metabolism (Alenghat et al., 2011; Chen et al. 2015; Gréchez-Cassiau et al., 2015). Genome-wide RNA expression profiling has shown that about $5 \%$ to $20 \%$ of the transcriptome exhibits circadian rhythmicity, and the oscillating genes vary in the different tissues of the organism, reaching the highest percentage in the liver, a vital organ that handles several functions, such as metabolism of glucose and lipids, protein synthesis anddetoxification of xenobiotics (Gachon et al., 2004; Gorné et al., 2015; Mazzoccoli et al., 2012c; Paredes et al., 2014). The basic mechanisms of functioning of the circadian molecular clock have been explored by studies using human cell lines and mouse genetic models (Lowrey \& Takahashi, 2011). Animal models are used for toxicology studies, but they do not address species-specific dissimilarities, so that human models are needed. Human cell culture models have been established to reduce the use of animals, and one of the favorite human in vitro models is represented by primary hepatocytes and hepatoma cell lines (such as HepG2). HepG2 cells are aneuploid and epithelial in morphology, derive from a well-differentiated hepatocellular carcinoma, but have a high degree of morphological and functional differentiation in vitro and render in vitro the polarized human hepatocytes (Wilkening et al., 2003). HepG2 cells are widely used as a model to study the molecular clockwork and in particular the hepatic circadian clock as well as downstream target genes (Chauvet et al., 2011; Crumbley et al., 2010; Gréchez-Cassiau et al., 2015; Lee et al., 2012; Matsunaga et al., 2012; Tao et al., 2011; Wang et al., 2010; Wang et al., 2013).

We aimed to investigate the molecular clockwork in two different experimental settings, in an hepatoma cell line (HepG2) in vitro and in mouse liver in vivo in order to evaluate if the patterns of periodicity and dynamics of clock gene expression are different in isolated cell aggregates when compared to the whole tissue using a new mathematical approach (Mazzoccoli et al., 2011; Mazzoccoli et al., 2012a). We compared the circadian trend of clock gene expression at the organ level, using previously reported data obtained in wild type mouse livers (Liu et al., 2007) and at the cellular level using new in vitro data obtained in a human hepatoma cell line.

\section{Materials and methods}

\section{Cell culture and serum shock procedures}

HepG2 cells were cultured in DMEM medium supplemented with $10 \%$ fetal bovine serum (FBS) and $1 \%$ of PEN/STREP (penicillin and streptomycin) at $37^{\circ} \mathrm{C}$ in $5 \% \mathrm{CO}_{2}$ atmosphere (Invitrogen Life Technologies, Milan, Italy). Cells were synchronized by serum shock performed as already described (Balsalobre et al., 1998); briefly $5 \times 10^{5}$ cells/6-wells were plated the day before the experiments. On the day of the experiments, culture medium was substituted with medium containing $50 \%$ of FBS for 2 hours and then replaced with the serum-free Dulbecco's Modified Eagle Medium (DMEM) (Balsalobre et al., 1998). The cells were then harvested every 3 hours for 28 hours after the serum shock. 


\section{Animals}

Animal experiments were performed as described elsewhere (Liu et al., 2007; Mazzoccoli et al., 2012a). We report previous data obtained in wild type mouse livers (Liu et al., 2007). C57BL/6 male mice aged 8 weeks were caged separately on a controlled 12 hour light (L)-dark (D) cycle (lights on at 08:00 hours), with ad libitum supply of food and water, 14 days after coming at the animal service. Animal care agreed with ethical criteria and guidelines of the Capital Medical University, Beijing, P. R. China. The experimental protocol conformed to international ethical standards (Portaluppi et al., 2010). Mice were sacrificed at intervals of 4 hours for 24 hours starting at 09:00 hours a.m. Livers were collected and preserved in RNAlater (Qiagen, Alameda, CA, USA). Total RNA was taken out from organs using RNAeasy kit (Qiagen, Alameda, CA, USA) following the manufacturer's instructions. To get rid of genomic DNA, total RNA was treated with RQ1 RNase-free DNase I (Promega, San Luis Obispo, CA, USA) following the manufacturer's instructions.

\section{Quantitative real-time polymerase chain reaction}

The procedures performed for processing animal tissues and carry out quantitative real-time polymerase chain reaction (qRT-PCR) are described elsewhere (Liu et al., 2007; Mazzoccoli et al., 2012a). Primer sequences used in qRT-PCR assay on mRNA extracted from mouse liver are showed in Table 1.

Table 1. Primer sequences used in qRT-PCR assay on mRNA extracted from mouse liver.

\begin{tabular}{ll}
\hline Bmal1 & Forward ACATAGGACACCTCGCAGAA, Reverse \\
Clock & AACCATCGACTTCTAGCGT \\
& Forward CCTATCCTACCTTCGCCACACA Reverse \\
Cry1 & TCCCGTGGAGAACCTAGAT \\
& Forward TTGCCTGTTCCTGACTCGT, Reverse \\
Cry2 & GACAGCCACATCCAACTTCC \\
& Forward TCGGCTCAACATTGAACGAA, Reverse \\
Per1 & GGGCCACTGGATAGTGTCT \\
& Forward CATGACTGCACTTCGGAGC, Reverse \\
Per2 & CTGACACAGGCAGAGCGTA \\
& Forward GGCTTCACCATGCCTGTGT, Reverse \\
NR1D1 & GGAGTTATTCGGAGGCAAGTGT \\
& Forward CGTCGCATCAATCGCAACC, Reverse \\
185 & GATGTGGAGTAGGTGAGGTC \\
& Forward TTCGGAACTGAGGCCATGAT, Reverse \\
& TTTCGCTCTGGTCCGTCTTG \\
\hline
\end{tabular}

In the in vitro study, qRT-PCR was performed on two biological replicates each assayed in triplicate as already reported (Benegiamo et al., 2013). Briefly, total RNA was extracted using the RNeasy® Mini Kit (Qiagen, Milan, Italy) from HepG2 cells harvested every 3 hours after serum shock at the indicated time points. One-step qRT-PCR was then performed using QuantiFast Sybr Green PCR kit following the manufactures' instructions (Qiagen, Milan, Italy). The following SYBR Green QuantiTect primers used for qRT-PCR were all purchased from Qiagen: Arntl (QT00068250), Clock (QT00054481), Cry1 (QT00025067), Cry2 (QT00094920) Per1 (QT00069265), Per2 (QT00011207) and NR1D1 (encoding Rev-Erba) (QT00000413). The expression levels of target gene were normalized to the housekeeping control gene TBP (encoding TATA binding protein) (QT00000721) (Qiagen, Milan, Italy), and data analysis was performed using SDS software (version 1.9.1; Applied Biosystems, Foster City, CA).

\section{Statistics}

To evaluate the periodicity of gene expression, time series of mRNA levels were normalized to percent of maximum and analyzed by Cosinor method as described elsewhere (Liu et al., 2007; Mazzoccoli et al., 2012a). To assess the dynamical patterns of gene expression, the slope of the cosine curve superimposed on raw data $(M=\Delta y / \Delta x)$, i.e. the derivative of gene expression level $(y)$ with respect to time $(x)$, defined as rate of change (RoC), was calculated between $90 \%$ of full amplitude and $10 \%$ of full amplitude of gene expression values $(R=90 \% A-10 \% A)$ as depicted in Figure 1 and according to the formula:

$$
\mathrm{RoC}=\frac{90 \% A-10 \% A}{t_{2}-t_{1}}
$$

Results are expressed as mean \pm standard error (SE). Data of gene expression were verified by normality test and equal variance test. Values were compared by means of the Student's $t$ test or Mann Whitney rank sum test as appropriate. Differences were considered statistically significant if $p \leq 0.05$. Statistical analyses were performed and graphs were drafted using the MATLAB statistical package (MathWorks, Natick, MA, USA). 


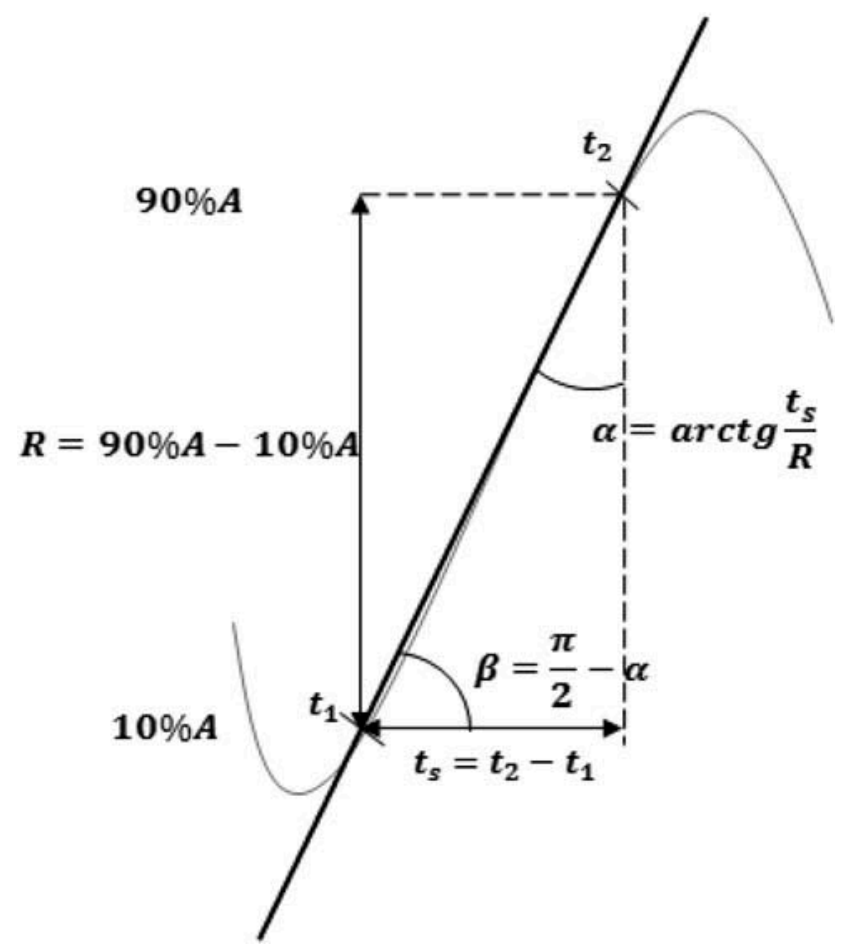

Figure 1. Scheme rendering the method used for computing the slope of the cosine curve superimposed on raw data of clock gene expression level.

\section{Results}

\section{Rhythm features of clock gene expression in HepG2 cells and mouse liver}

Circadian gene expression was investigated in synchronized HepG 2 cells harvested every 3 hours for 28 hours after serum shock, and in the liver of mice housed on a 12-hour L-D cycle and sacrificed at 4-hour intervals for 24 hours. Rhythm features of clock gene expression in HepG2 cells and liver were evaluated through the Cosinor method. Results from 24-hour Cosinor analyses for circadian time effects on original values of expression levels for the examined clock gene in HepG2 cells and mouse liver are listed in Table 2 and depicted in Figures 2-5. A significant 24-hour rhythmic component was found for five clock genes in the HepG2 cells (Bmal1, Cry1,Per1, Per2, NR1D1) and for six clock genes in the mouse liver (Bmal1, Clock, Cry1, Per1, Per2, NR1D1). The amplitude of oscillation as rendered by the cosine curve was greater in mouse liver than in HepG2 cells for Bmal1, Clock, Cry2, Per1, Per2 and NR1D1, while the phase of oscillation was significantly different for Bmal1, Cry1, Per1 and
Table 2. Rhythm features of clock gene expression in HepG2 cells and mouse liver $( \pm \mathrm{SE})$.

\begin{tabular}{|c|c|c|c|c|}
\hline & MESOR & Amplitude & Acrophase & $\begin{array}{c}p \\
\text { value }^{\#}\end{array}$ \\
\hline \multicolumn{5}{|c|}{ HepG2 cells } \\
\hline Bmal1 & $0.512 \pm 0.01$ & $0.171 \pm 0.03$ & $11: 34 \pm 01: 06$ hours & 0.030 \\
\hline Clock & $0.436 \pm 0.01$ & $0.265 \pm 0.02$ & $18: 26 \pm 00: 59$ hours & 0.133 \\
\hline Cry1 & $0.484 \pm 0.01$ & $0.291 \pm 0.01$ & $10: 49 \pm 01: 23$ hours & 0.067 \\
\hline Cry2 & $0.507 \pm 0.03$ & $0.287 \pm 0.02$ & $11: 52 \pm 01: 40$ hours & 0.045 \\
\hline Per1 & $0.421 \pm 0.02$ & $0.187 \pm 0.01$ & $21: 42 \pm 00: 42$ hours & 0.034 \\
\hline Per2 & $0.513 \pm 0.01$ & $0.251 \pm 0.02$ & $14: 31 \pm 01: 12$ hours & 0.007 \\
\hline NR1D1 & $0.244 \pm 0.02$ & $0.281 \pm 0.03$ & $11: 14 \pm 02: 01$ hours & 0.027 \\
\hline \multicolumn{5}{|c|}{ Mouse liver } \\
\hline Bmal1 & $0.483 \pm 0.01$ & $0.512 \pm 0.02^{*}$ & $20: 52 \pm 02: 27$ hours * & 0.019 \\
\hline Clock & $0.743 \pm 0.04^{*}$ & $0.310 \pm 0.01^{*}$ & $20: 55 \pm$ 02:01 hours & 0.251 \\
\hline Cry1 & $0.432 \pm 0.01$ & $0.313 \pm 0.02$ & $18: 11 \pm 01: 12$ hours * & 0.112 \\
\hline Cry2 & $0.711 \pm 0.02^{*}$ & $0.377 \pm 0.01^{*}$ & $13: 10 \pm 01: 43$ hours & 0.015 \\
\hline Per1 & $0.419 \pm 0.03$ & $0.219 \pm 0.01^{*}$ & $11: 07 \pm 01: 35$ hours $*$ & 0.011 \\
\hline Per2 & $0.516 \pm 0.02$ & $0.454 \pm 0.01^{*}$ & $14: 09 \pm 01: 30$ hours & 0.006 \\
\hline NR1D1 & $0.328 \pm 0.01^{*}$ & $0.480 \pm 0.03^{*}$ & $5: 42 \pm 00: 19$ hours $*$ & 0.010 \\
\hline \multicolumn{5}{|c|}{$\begin{array}{l}\text { H value for statistical significance of parameter estimates: rhythm } \\
\text { detection was considered statistically significant when } p<0.05 ;{ }^{*} p \\
<0.05 \text { for hypotheses regarding differences among the values com- } \\
\text { pared by means of the Student's } t \text { test or Mann Whitney rank sum } \\
\text { test as appropriate; SE = standard error. }\end{array}$} \\
\hline
\end{tabular}

NR1D1. The comparison of phase relationships among multiple clock genes evidenced that in mouse liver there was a proper pattern of expression, with phase correspondence for Bmall and Clock as well as for period and cryptochrome genes, and opposing phase for Bmall and NR1D1. In HepG2 cells, Clock expression was delayed respect to Bmall expression, which showed a peak synchronous with Cry1, Cry2 and NR1D1 expression, while Per2 phase was advanced respect to Per1 expression.

\section{Rate of change (RoC) of clock gene expression levels in HepG2 cells and mouse liver}

Results from comparison of $\mathrm{RoC}$ of clock gene expression in HepG2 cells and mouse liver are listed in Table 3 and depicted in Figure 6. A statistically significant difference was evidenced for the RoC of Bmal1, Per1, Per2 and NR1D1 mRNA expression level, which was greater in mouse liver.

\section{Discussion}

Circadian rhythmicity of mammalian biological phenomena is generated by genetically encoded biological clocks ticking through feedback loops 

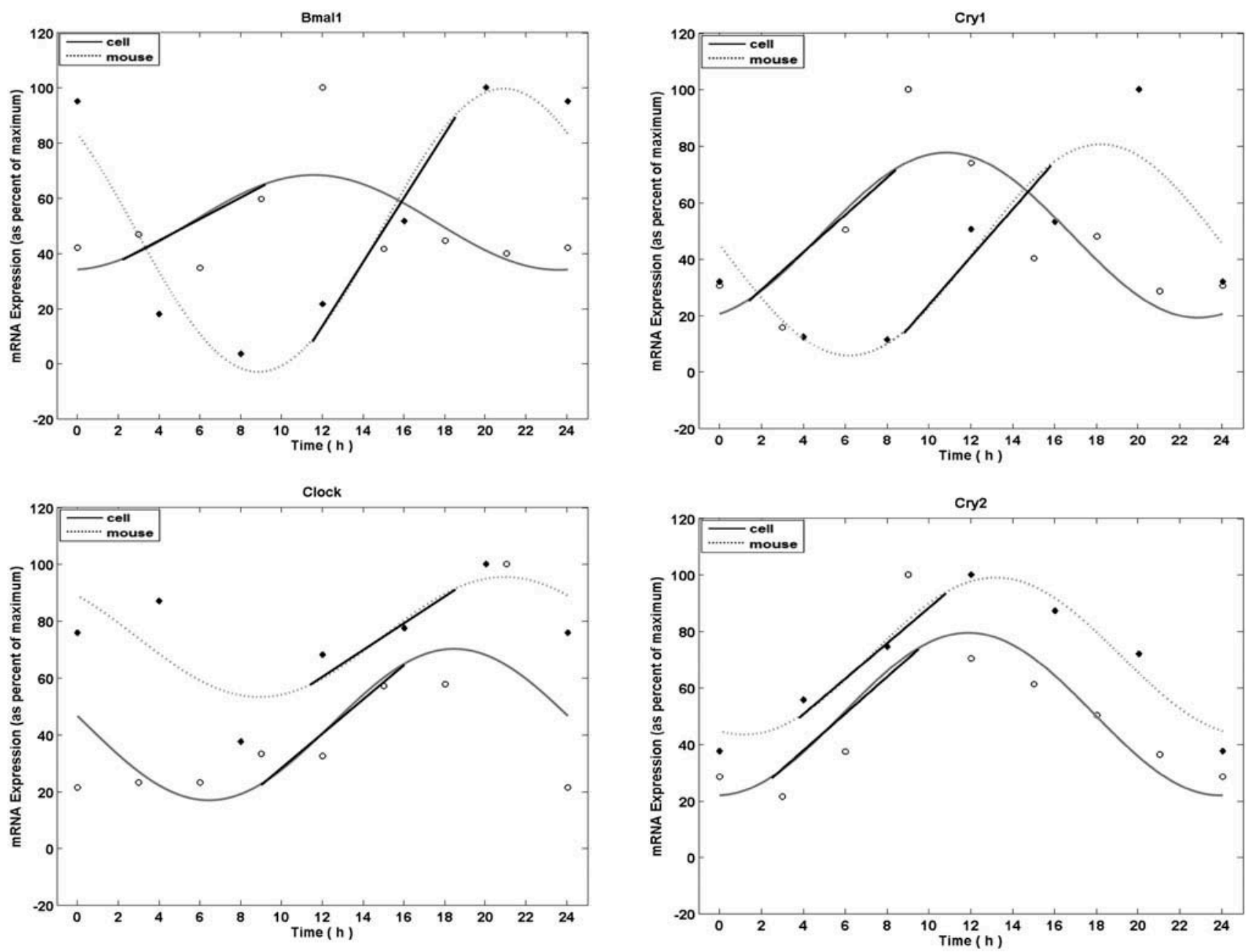

Figure 2. Time-related patterns of Bmal1 and Clock expression in wild type mouse liver and in a human hepatoma cell line. $x-y$ plots representing from top to bottom the fitted cosine curves (continuous and dotted lines) superimposed on raw data (circle and rhombus) of clock gene expression level in liver tissue from mice ( $n=3$ per time point) sacrificed at 4-hour intervals for 24 hours (data obtained in wild type mouse livers from Liu et al., 2007) and HepG2 cells synchronized after serum shock and collected at 3-hour intervals for 28 hours.

that regulate the circadian genetic transcription. In any single cell, basic functions are driven by interacting positive and negative limbs in which the expression of clock genes is suppressed periodically by their protein products (Dibner et al., 2010; Ueda et al., 2005). Accordingly, in our study the mRNA expression levels of the clock genes examined in HepG2 cells showed time-qualified variation, characterized by statistically significant 24 hour periodicity for Per1, Per2, Cry1, Bmal1 and NR1D1. The biological oscillators are synchronized by the central pacemaker located in the $\mathrm{SCN}$, which via circadian output pathways drives

Figure 3. Time-related patterns of Cry1 and Cry2 expression in wild type mouse liver and in a human hepatoma cell line. $x-y$ plots representing from top to bottom the fitted cosine curves (continuous and dotted lines) superimposed on raw data (circle and rhombus) of clock gene expression level in liver tissue from mice ( $n=3$ per time point) sacrificed at 4-hour intervals for 24 hours (data obtained in wild type mouse livers from Liu et al., 2007) and HepG2 cells synchronized after serum shock and collected at 3-hour intervals for 28 hours.

the autonomous clocks in all peripheral tissues. We have evaluated clock gene expression patterns in hepatic tissue of an animal model using a data subset (Liu et al., 2007). A significant 24-hour rhythmic component was found for Bmal1, Clock, Cry1, Per1, Per2 and NR1D1 expression in the mouse liver.

The circadian genes Bmal1, Clock, Cry2, Per1, Per2 and NR1D1 showed greater amplitude of oscillation in mouse liver than in HepG2 cells, while the phase of oscillation was significantly different for Bmal1, Cry1, Per1 and NR1D1. The phase relationships among clock gene expression 

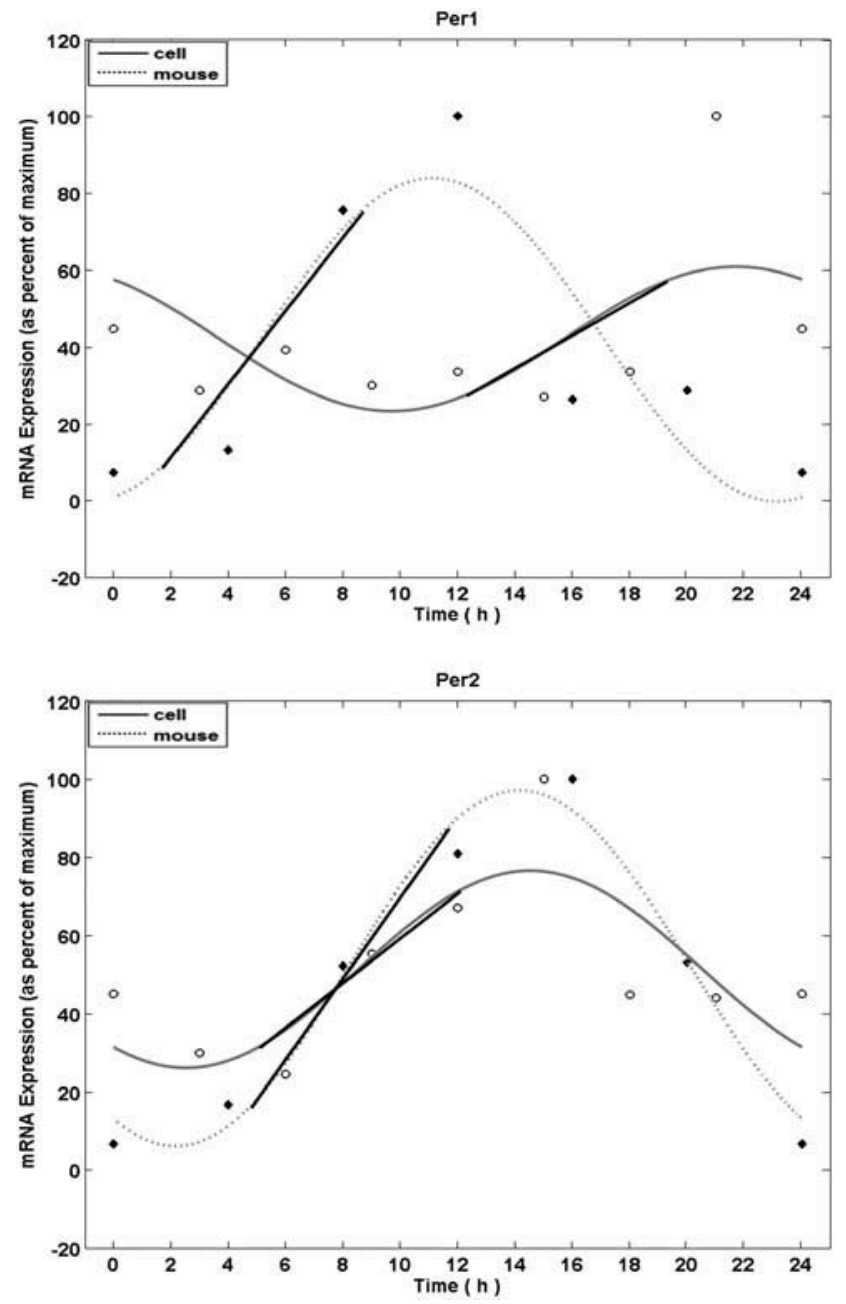

Figure 4. Time-related patterns of Per1 and Per2 expression in wild type mouse liver and in a human hepatoma cell line. $x-y$ plots representing from top to bottom the fitted cosine curves (continuous and dotted lines) superimposed on raw data (circle and rhombus) of clock gene expression level in liver tissue from mice ( $n=3$ per time point) sacrificed at 4-hour intervals for 24 hours (data obtained in wild type mouse livers from Liu et al., 2007) and HepG2 cells synchronized after serum shock and collected at 3-hour intervals for 28 hours.

showed a proper pattern in mouse liver, whereas in the hepatoma cell line the serial activation of transcription was similar to previous results (Crumbley et al., 2010) and deranged respect to the appropriate outline (Takahashi, 2015; UkaiTadenuma et al., 2011). In particular, Bmall expression, advanced respect to Clock expression, was synchronous with phase expression of Cry1, Cry2 and above all NR1D1, the gene encoding the transcription factor Rev-erba.

Rev-erba propels the magnitude and strength of oscillation in the molecular clockwork through control of Bmal1 and Clock rhythmic

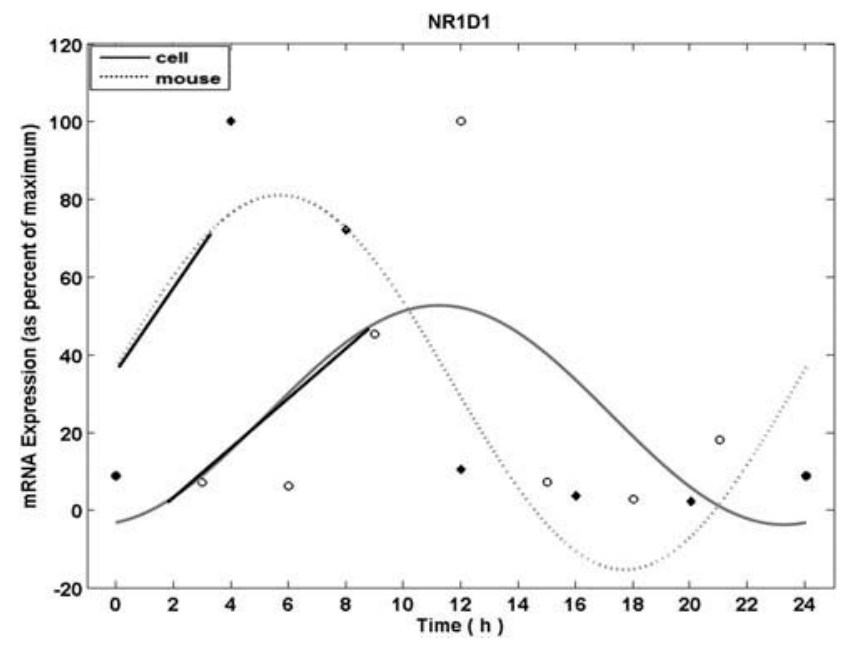

Figure 5. Time-related patterns of NR1D1 expression in wild type mouse liver and in a human hepatoma cell line. $x-y$ plots representing from top to bottom the fitted cosine curves (continuous and dotted lines) superimposed on raw data (circle and rhombus) of clock gene expression level in liver tissue from mice ( $n=3$ per time point) sacrificed at 4-hour intervals for 24 hours (data obtained in wild type mouse livers from Liu et al., 2007) and HepG2 cells synchronized after serum shock and collected at 3-hour intervals for 28 hours.

Table 3. Rate of change (\%) of clock gene mRNA expression in HepG2 cells and mouse liver $( \pm$ SE).

\begin{tabular}{lcl}
\hline & HepG2 cells & Mouse liver \\
\hline Bmal1 & $1.91 \pm 0.2$ & $4.27 \pm 0.4^{*}$ \\
Clock & $1.56 \pm 0.1$ & $1.76 \pm 0.2$ \\
Cry1 & $3.25 \pm 0.3$ & $3.11 \pm 0.3$ \\
Cry2 & $2.51 \pm 0.4$ & $2.31 \pm 0.1$ \\
Per1 & $1.47 \pm 0.2$ & $3.52 \pm 0.2^{*}$ \\
Per2 & $1.77 \pm 0.1$ & $3.86 \pm 0.3^{*}$ \\
NR1D1 & $2.05 \pm 0.3$ & $4.01 \pm 0.4^{*}$ \\
\hline
\end{tabular}

${ }^{*} p<0.05$ for hypotheses regarding differences among the values compared by means of the Student's $t$ test or Mann Whitney rank sum test as appropriate; $\mathrm{SE}=$ standard error.

transcription, and in our study its expression showed a clear circadian rhythmicity considering both experimental settings. A statistically significant difference was found comparing the RoC of expression of the clock genes showing circadian rhythmicity of mRNA level variation, with higher levels found for Bmal1, Per1, Per2 and NR1D1 in mouse liver. Data obtained from our study suggest that the periodicity of clock gene expression is similar in the two experimental settings when comparing time-qualified mRNA levels in HepG2 cells synchronized by serum shock and in the hepatic tissue of mice housed on a 12 -hour L-D cycle, highlighting the similarity of the two experimental models when exploring the 


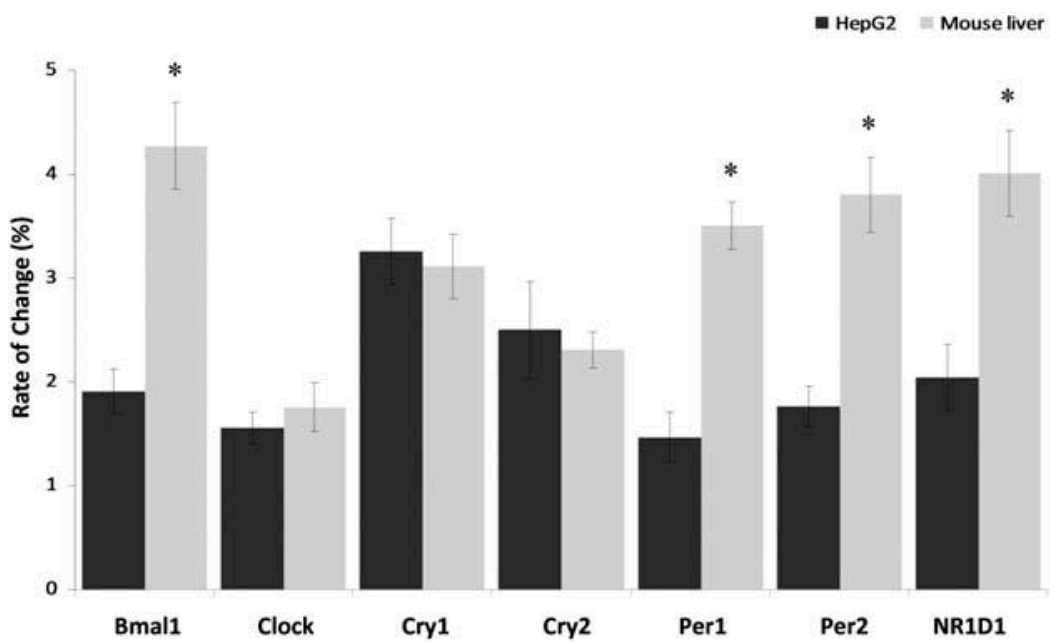

Figure 6. Rate of change $(\mathrm{RoC})$ of clock gene expression levels in wild type mouse liver and in a human hepatoma cell line. Comparison of the derivative of gene expression level in HepG2 cells and mouse liver with respect to time calculated between zenith and nadir gene expression; ${ }^{*} p<0.05$.

periodicity of function of the circadian clockwork. Anyway, the complexity of the animal model allows to explore more complete scenarios and some studies can be performed only with mice (i.e. sleep disorders, behavior derangements). Accordingly, in our study the liver tissue is characterized by higher amplitudes and stronger dynamics of clock gene expression, probably in relationship to the effect of local and systemic cues, which strengthen the coupling between the single elements in a coupled oscillator array. In the liver, the transcriptional activity is principally directed by the light-driven SCN through autonomic innervation (Cailotto et al., 2009), but another powerful entraining factor is represented by metabolic fluxes related to feeding (Damiola et al., 2000; Stokkan et al., 2001). Transcription of genes involved in metabolic processes is easily decoupled when food intake is desynchronized from normal behavioral cycles of sleep/wake, rest/activity, fasting/feeding dictated by the SCN, and regularly scheduled daily meals become the dominant entraining stimulus (Hoogerwerf et al., 2007; Mazzoccoli et al., 2012d; Polidarová et al., 2011; Vollmers et al., 2009). Rev-Erba and other nuclear receptors are expressed in metabolically active tissues, such as liver, and play a key role in gauging nutrients and linking energy flux with transcriptional activity (Burris, 2008). Accordingly, in the animal model, NR1D1 showed the strongest oscillation among the clock genes characterized by circadian rhythmicity of expression. Great amplitude of oscillation characterized NR1D1 expression also in vitro, suggesting that the serum shock may represent a metabolic input over and above an osmotic challenge in the synchronization of the cultured HepG2 cells.

Experiments performed in vitro take advantage of a controlled environment outside of a living being, but they fall short in fully reproducing the exact cellular conditions of an organism. For instance, in culture-grown cellular systems, it is feasible to evaluate time-series analysis of gene expression, but protein expression as well as concentrations of molecules competing for nuclear receptors are not entirely similar to what is found in the whole organism. Besides, another factor influencing the pattern of gene expression in cell cultures is represented by cell density: PER2:LUC bioluminescence circadian rhythmicity was significantly decreased in low-density fibroblast cultures, most likely in relation to the absence of either constitutive or rhythmic paracrine signals from neighboring fibroblasts, suggesting that rhythmic gene expression requires not necessarily rhythmic paracrine signals from adjacent cells (Noguchi et al., 2013). In its side, experiments employing an entire animal model allow to explore the time-related patterns of gene expression and are more appropriate for observing the overall effects on a living being. In humans, 
it is very difficult to carry out studies that provide time-series analysis of gene expression at the tissue level. This approach was performed through timed serial biopsies of bone marrow (Tsinkalovsky et al., 2007) and adipose tissue (Loboda et al., 2009) to evaluate time-related patterns of clock gene transcription in tissues that are accessible for sample collection. Another approach used was to perform skin biopsies from individual subjects for fibroblasts to be cultivated in vitro to measure clock gene expression and compare the period length of the in vitro rhythm (in vitro period) with the intrinsic circadian period $\tau$ (in vivo period) assessed by means of forced desynchrony protocol, circadian/sleep parameters evaluated by questionnaires, sleep log and actigraphy as well as the secretion pattern of the hormone melatonin to characterize circadian phenotypes (Hasan et al., 2012; Hida et al., 2013; Pagani et al., 2010). On the contrary, this kind of study is not feasible on human liver tissue, although information on the dynamics of gene transcription that could be obtained would be of great importance to investigate physiological processes and pathological mechanisms of disease. As stated above, HepG2 cells are widely used for analysis of the molecular clockwork in human liver, but a comparison with the dynamics of expression evaluated at the tissue level is not possible, and for this reason we compared them with mouse liver as tissue model. From the comparison, it comes out that only the periodicity is similar between in vitro and in vivo models, while the dynamics of expression (MESOR, amplitude, phase, slope) are greatly different.

In conclusion, the clock gene machinery shows similar periodicity of expression but different dynamics of transcription when in vitro and animal models are used to explore the biological clock, suggesting that in comparison to cultured hepatic cells, in the hepatic tissue dynamical patterns of circadian gene expression are greatly different, probably in relation to oscillator coupling and systemic or local cues. The results of our chronobiological analysis, performed by using biological materials "Of Mice and Men," as the Nobel Prize-winning author John Steinbeck titled is novel, corroborate the evidence that, as an alternative to animal experiments, cultured human hepatic cell lines are useful and valuable tools as scientific investigational models to evaluate only the periodicity of functioning of the molecular clockwork, but fall short in reproducing the dynamics assessable at the tissue level.

\section{Acknowledgements}

We thank Valerio Pazienza for technical support and Massimo Francavilla for providing support in computer-based analyses.

\section{Funding}

The study was supported by the " $5 \times 1000$ " voluntary contribution, by a grant from the Italian Ministry of Health to GM (RC1201ME04, RC1203ME46, RC1302ME31, RC1403ME50 and RC1504ME53) through Department of Medical Sciences, Division of Internal Medicine and Chronobiology Unit, IRCCS Scientific Institute and Regional General Hospital "Casa Sollievo della Sofferenza”, Opera di Padre Pio da Pietrelcina, San Giovanni Rotondo (FG), Italy, and by a grant to MV from the Italian Association on Cancer Research (AIRC) (MFAG 2012-13419).

\section{Declaration of interest statement}

The authors declare that there are no conflicts of interest with respect to the authorship and/or publication of this article.

\section{ORCID}

Gianluigi Mazzoccoli (D) http://orcid.org/0000-0003-35357635

\section{References}

Agostino PV, Harrington ME, Ralph MR, Golombek DA. (2009). Casein kinase-1-epsilon (CK1epsilon) and circadian photic responses in hamsters. Chronobiol Int. 26:126-33.

Alenghat T, Meyers K, Mullican SE, et al. (2011). Crosstalk between components of circadian and metabolic cycles in mammals. Cell Metab. 13:125-37.

Balsalobre A, Damiola F, Schibler U. (1998). A serum shock induces circadian gene expression in mammalian tissue culture cells. Cell. 93:929-37.

Bell-Pedersen D, Cassone VM, Earnest DJ, et al. (2005). Circadian rhythms from multiple oscillators: Lessons from diverse organisms. Nat Rev Genet. 6:544-56.

Benegiamo G, Mazzoccoli G, Cappello F, et al. (2013). Mutual antagonism between circadian protein period 2 and hepatitis $\mathrm{C}$ virus replication in hepatocytes. PLoS One. 8(4):e60527.

Burris TP. (2008). Nuclear hormone receptors for heme: REV-ERBalpha and REV-ERBbeta are ligand-regulated 
components of the mammalian clock. Mol Endocrinol. 22:1509-20.

Cailotto C, Lei J, van der Vliet J, et al. (2009). Effects of nocturnal light on (clock) gene expression in peripheral organs: A role for the autonomic innervation of the liver. PLoS One. 4(5):e5650.

Chauvet C, Vanhoutteghem A, Duhem C, et al. (2011). Control of gene expression by the retinoic acid-related orphan receptor alpha in HepG2 human hepatoma cells. PLoS One. 6(7):e22545.

Chen H, Isayama K, Kumazawa M, et al. (2015). Integration of the nuclear receptor REV-ERBa linked with circadian oscillators in the expressions of Alas1, Ppargc1a, and Il6 genes in rat granulosa cells. Chronobiol Int. 32(6):739-49.

Cho H, Zhao X, Hatori M, et al. (2012). Regulation of circadian behaviour and metabolism by REV-ERB- $\alpha$ and REV-ERB- $\beta$. Nature. 485:123-27.

Crumbley C, Wang Y, Kojetin DJ, Burris TP. (2010). Characterization of the core mammalian clock component, NPAS2, as a REV-ERBalpha/RORalpha target gene. J Biol Chem. 285:35386-92.

Damiola F, Le Minh N, Preitner N, et al. (2000). Restricted feeding uncouples circadian oscillators in peripheral tissues from the central pacemaker in the suprachiasmatic nucleus. Genes Dev. 14: 2950-61.

Dibner C, Schibler U, Albrecht U. (2010). The mammalian circadian timing system: Organization and coordination of central and peripheral clocks. Ann Rev Physiol. 72:517-49.

Duguay D, Cermakian N. (2009). The crosstalk between physiology and circadian clock proteins. Chronobiol Int. 26:1479-513.

Eide EJ, Vielhaber EL, Hinz WA, Virshup DM. (2002). The circadian regulatory proteins BMAL1 and cryptochromes are substrates of casein kinase Ic. J Biol Chem. 277:17248-54

Gachon F, Nagoshi E, Brown SA, et al. (2004). The mammalian circadian timing system: From gene expression to physiology. Chromosoma. 113:103-12.

Gorné LD, Acosta-Rodríguez VA, Pasquaré SJ, et al. (2015). The mouse liver displays daily rhythms in the metabolism of phospholipids and in the activity of lipid synthesizing enzymes. Chronobiol Int. 32(1):11-26.

Gréchez-Cassiau A, Feillet C, Guérin S, Delaunay F. (2015). The hepatic circadian clock regulates the choline kinase $\alpha$ gene through the BMAL1-REV-ERBa axis. Chronobiol Int. 32(6):774-84.

Hasan S, Santhi N, Lazar AS, et al. (2012). Assessment of circadian rhythms in humans: Comparison of real-time fibroblast reporter imaging with plasma melatonin. FASEB J. 26(6):2414-23.

Herzog ED. (2007). Neurons and networks in daily rhythms. Nat Rev Neurosci. 8:790-802.

Hida A, Kitamura S, Ohsawa Y, et al. (2013). In vitro circadian period is associated with circadian/sleep preference. Sci Rep. 3:2074.

Hoogerwerf WA, Hellmich HL, Cornélissen G, et al. (2007). Clock gene expression in the murine gastrointestinal tract:
Endogenous rhythmicity and effects of a feeding regimen. Gastroenterology. 133:1250-60.

Lee YJ, Han DH, Pak YK, Cho SH. (2012). Circadian regulation of low density lipoprotein receptor promoter activity by CLOCK/BMAL1, Hes1 and Hes6. Exp Mol Med. 44:642-52.

Liu S, Cai Y, Sothern RB, et al. (2007). Chronobiological analysis of circadian patterns in transcription of seven key clock genes in six peripheral tissues in mice. Chronobiol Int. 24(5):793-820.

Loboda A, Kraft WK, Fine B, et al. (2009). Diurnal variation of the human adipose transcriptome and the link to metabolic disease. BMC Med Genomics. 2:7.

Lowrey PL, Takahashi JS. (2011). Genetics of circadian rhythms in Mammalian model organisms. Adv Genet 74:175-230.

Matsunaga N, Inoue M, Kusunose N, et al. (2012). Timedependent interaction between differentiated embryo chondrocyte- 2 and CCAAT/enhancer-binding protein a underlies the circadian expression of CYP2D6 in serumshocked HepG2 cells. Mol Pharmacol. 81(5):739-47.

Mazzoccoli G. (2011). The timing clockwork of life. J Biol Regul Homeost Agents. 25:137-43.

Mazzoccoli G, Sothern RB, Greco G, et al. (2011). Timerelated dynamics of variation in core clock gene expression levels in tissues relevant to the immune system. Int J Immunopathol Pharmacol. 24:869-79.

Mazzoccoli G, Francavilla M, Giuliani F, et al. (2012a). Clock gene expression in mouse kidney and testis: Analysis of periodical and dynamical patterns. J Biol Regul Homeost Agents. 26:303.

Mazzoccoli G, Cai Y, Liu S, et al. (2012b). REV-ERBalpha and the clock gene machinery in mouse peripheral tissues: A possible role as a synchronizing hinge. J Biol Regul Homeost Agents. 26:265.

Mazzoccoli G, Pazienza V, Vinciguerra M. (2012c). Clock genes and clock controlled genes in the regulation of metabolic rhythms. Chronobiol Int. 29:227-51.

Mazzoccoli G, Francavilla M, Pazienza V, et al. (2012d). Differential patterns in the periodicity and dynamics of clock gene expression in mouse liver and stomach. Chronobiol Int. 29:1300-11.

Nagoshi E, Saini C, Bauer C, et al. (2004). Circadian gene expression in individual fibroblasts: Cell-autonomous and self-sustained oscillators pass time to daughter cells. Cell. 119:693-705.

Noguchi T, Wang LL, Welsh DK. (2013). Fibroblast PER2 circadian rhythmicity depends on cell density. J Biol Rhythms. 28(3):183-92.

Pagani L, Semenova EA, Moriggi E, et al. (2010). The physiological period length of the human circadian clock in vivo is directly proportional to period in human fibroblasts. PLoS One. 5(10):e13376.

Paredes JF, Vera LM, Martinez-Lopez FJ, et al. (2014). Circadian rhythms of gene expression of lipid metabolism in Gilthead Sea bream liver: Synchronisation to light and feeding time. Chronobiol Int. 31(5):613-26. 
Polidarová L, Sládek M, Soták M, et al. (2011). Hepatic, duodenal, and colonic circadian clocks differ in their persistence under conditions of constant light and in their entrainment by restricted feeding. Chronobiol Int. 28:204-15.

Portaluppi F, Smolensky MH, Touitou Y. (2010). Ethics and methods for biological rhythm research on animals and human beings. Chronobiol Int. 27:1911-29.

Stokkan KA, Yamazaki S, Tei H, et al. (2001). Entrainment of the circadian clock in the liver by feeding. Science. 291:490-3.

Takahashi JS, Hong HK, Ko CH, McDearmon EL. (2008). The genetics of mammalian circadian order and disorder: Implications for physiology and disease. Nat Rev Genet. 9:764-75.

Takahashi JS. (2015). Molecular components of the circadian clock in mammals. Diabetes Obes Metab 17 (Suppl 1):6-11.

Tao W, Chen S, Shi G, et al. (2011). SWItch/sucrose nonfermentable (SWI/SNF) complex subunit BAF60a integrates hepatic circadian clock and energy metabolism. Hepatology. 54:1410-20.

Tsinkalovsky O, Smaaland R, Rosenlund B, et al. (2007). Circadian variations in clock gene expression of human bone marrow CD34+ cells. J Biol Rhythms. 22 (2):140-50.

Ueda UR, Hayashi S, Chen W, et al. (2005). System-level identification of transcriptional circuits underlying mammalian circadian clocks. Nat Genet. 37:187-92.

Ukai-Tadenuma M, Yamada RG, Xu H, et al. (2011). Delay in feedback repression by cryptochrome 1 is required for circadian clock function. Cell. 144:268-81.

Vollmers C, Gill S, DiTacchio L, et al. (2009). Time of feeding and the intrinsic circadian clock drive rhythms in hepatic gene expression. Proc Natl Acad Sci USA. 106 (50):21453-8.

Wang Y, Solt LA, Burris TP. (2010). Regulation of FGF21 expression and secretion by retinoic acid receptorrelated orphan receptor alpha. J Biol Chem. 285 (21):15668-73.

Wang T, Yang P, Zhan Y, et al. (2013). Deletion of circadian gene Per1 alleviates acute ethanol-induced hepatotoxicity in mice. Toxicology. 314:193-201.

Wilkening S, Stahl F, Bader A. (2003). Comparison of primary human hepatocytes and hepatoma cell line Hepg2 with regard to their biotransformation properties. Drug Metab Dispos. 31:1035-42. 